\title{
Du modèle au territoire: le peuplement de la Suisse, à l'exemple de régions-test
}

\begin{abstract}
Résumé
Dans la tradition des études des systèmes urbains fondées sur la théorie des lieux centraux, certaines recherches ont mis l'accent sur la constance de l'indice de concentration dans un système d'une certaine taille et obéissant à l'un des trois grands "principes organisateurs" dégagés par la théorie de W. Christaller. Cette étude analyse un changement de structure, grâce à un test longitudinal sur la Suisse (18501990) laissant entrevoir le passage d'une territorialité agricole à une territorialité industrialo-urbaine, dans six régions représentatives, pour tenter une prévision.

Lieux centraux, structure hiérarchique, réseau urbain suisse.
\end{abstract}

\begin{abstract}
In the tradition of studies about central places theory in urban systems, some contributions have emphasized the invariability of the concentration index in systems of a certain size. that comply with one of the main organizing principles elucidated by W. Christaller. This research of the structural change is an empirical, longitudinal study of some Swiss representative regions (1850-1990), their transformation of agricultural land into industrial and urban-industrial territoriality, and a prevision of their future.
\end{abstract}

Central places, hierarchical structure, Swiss urban system.

\section{Zusammenfassung}

Im traditionellen Studium der urbanen Struktur nach der Theorie von W. Christaller (Die zentralen Orte in Süddeutschland, 1933) wurde meist der Akzent auf die Konzentrationsmerkmale des Systems nach dem Handels-, Transport- und Verwaltungsprinzip gelegt. In diesem Beitrag werden Veränderungen dieser Struktur aufgezeigt, basierend auf einer empirischen, thematischen Untersuchung über die Schweiz (1850-1990); und am Beispiel einiger typischen Regionen wird eine Prognose gewagt.

Zentrale Orte, hierarchische Struktur, Stadtsystem in der Schweiz.

\section{Introduction}

Le territoire helvétique présente une structure de peuplement caractérisée par des contrastes morphologiques, qui désigne quatre ensembles diagonaux classiques formés par le relief, ponctués d'une série de bassins littoraux. Cette donnée physique favorise l'émergence d'une organisation urbaine hiérarchisée, dont la dynamique montre une certaine invariance dans la longue durée. Le modèle proposé dans cette contribution repose sur une écogénèse en trois stades: la longue phase agricole, la phase d'articulation industrielle en réseaux de transport et le processus récent de restructuration urbaine à une échelle nationale et transnationale. L'accession de la Suisse à une nouvelle échelle de développement urbain, par la différenciation des rythmes de mutation territoriale sur un modèle global, incite à vouloir esquisser le développement territorial probable des deux prochaines décennies. L'analyse des différents rythmes régionaux à partir de l'âge d'or agricole (placé vers 1850) permet d'anticiper quelque peu le mouvement de sous-ensembles centraux et périphériques vers une intégration par diffusion de la centralité urbaine.

Dans la tradition des études des systemes urbains fondées sur la théorie des lieux centraux, certaines recherches ont mis l'accent sur la constance de l'indice de concentration dans un systeme d'une certaine taille et obéissant à l'un des trois grands "principes organisateurs» dégagés par la théorie de W. Christaller. L'hypothèse de travail qui guide ces recherches est un changement de structure, lié à une évolution des rapports territoriaux, et que mesure une variation de l'ISH (indice de structuration hiérarchique, cf. infra). Un test longitudinal sur la Suisse (1850-1990) laisse entrevoir les grands moments du passage d'une territorialité agricole à une territorialité industrielle et industrialo-urbaine, dans six régions représentatives d'une diversité de rythme de croissance; la carte thématique demeure, pour ce test, un incontournable instrument d'appoint.

\section{La mise au point d'un modèle de peuplement}

Dans tous les contextes connus de transformation territoriale, le rôle et la hiérarchie des centres évoluent en même temps que varie la taille démographique de chaque entité urbaine. Ainsi, l'exode rural observé dès le dixhuitième siècle, en Europe occidentale, n'a pas seulement enflé les bourgs, en sélectionnant ceux qui devaient déclencher les phases successives de la révolution industrielle; il a aussi redistribue tous les lieux centraux dans leur rang d'importance, selon une logique nouvelle, qui

Charles Hussy, PD, Dr, Université de Genève, Institut de Géographie, Uni-Mail, 102, boulevard Carl-Vogt, 1211 Genève 4 
privilégiait le transport (Figure I. Région de la Suisse centrale, 1850-1890, exode rural et montagnard). Le cas de la Suisse centrale se singularise par un effet de relief: tandis que l'exode se manifeste sur les deux versants nord et sud de la chaîne alpine (Préalpes lucernoises et vallée du Rhin supérieur), mais très peu, en revanche, sur les Alpes bernoises et glaronnaises, la concentration apparaît avec une même force dans les cantons alpins, au pied des grands massifs. Cette pénétration marque l'entrée de la modernité urbaine, par le chemin de fer et l'industrialisation, jusqu'au cœur du pays.

On ne saurait donc faire l'impasse sur la réflexion théorique ouverte par Christaller et Lösch à propos de ces phénomènes; il faut au contraire sans cesse revenir, en observant des situations empiriques, aux explications qu'ils nous proposent dans leur élémentaire simplicité. Si l'on souhaite, de surcroit, extrapoler des mutations territoriales, il s'agit en effet d'éprouver la validité de ces stades d'urbanisation dans une théorie de l'écogénèse (HussY, 1980) et de calibrer la mesure des territoires réels en fonction des invariants observés par la théorie. L'essai présenté se base sur quelques étapes de la recherche en ce domaine et propose un outil de mise en œuvre empirique de ses résultats actuels, puis de ceux prévisibles dans le futur.

La réflexion sur la structure des établissements humains passe par une phase géométrique, puis statistique; elle peut déboucher sur un indice structurel (HUSSY, MERCIER, RAFFESTIN, 1985), qui permet d'envisager une prévision.

1. Walter Christaller tire de l'observation de l'Allemagne du Sud l'idée d'un modele d'essence géométrique, que August Lösch formalise: $\mathrm{k}=3,4,7$. Le principe organisateur $(k)$, qui désigne selon nous un stade d'écogénèse, exprime la desserte de localités d'un certain niveau (ou taille), par une localité de niveau supérieur. Aux moments critiques de l'écogénèse, le nombre de dessertes augmente en fonction du renforcement de la centralité des villes et de leur taille, qui fait s'élever la concentration du système et augmenter l'étendue des systèmes (voir la Figure 2). Une large redistribution des rangs sopère alors, concrétisée par le déclin de bourgs importants à un stade antérieur et l'accession de petites nodosités à un rang supérieur. La substitution de rangs autour de la ville principale manifeste ainsi un branle-bas général, synonyme de changement profond de la territorialité. $\mathrm{Si}$, au contraire, le nombre de dessertes demeure identique dans le temps, autrement dit, la logique d'ensemble, de même que la hiérarchie des centres, un système peut néanmoins voir augmenter sa population par un accroissement du nombre de niveaux, qui lui aussi relève la concentration, tout en obéissant au même principe organisateur.

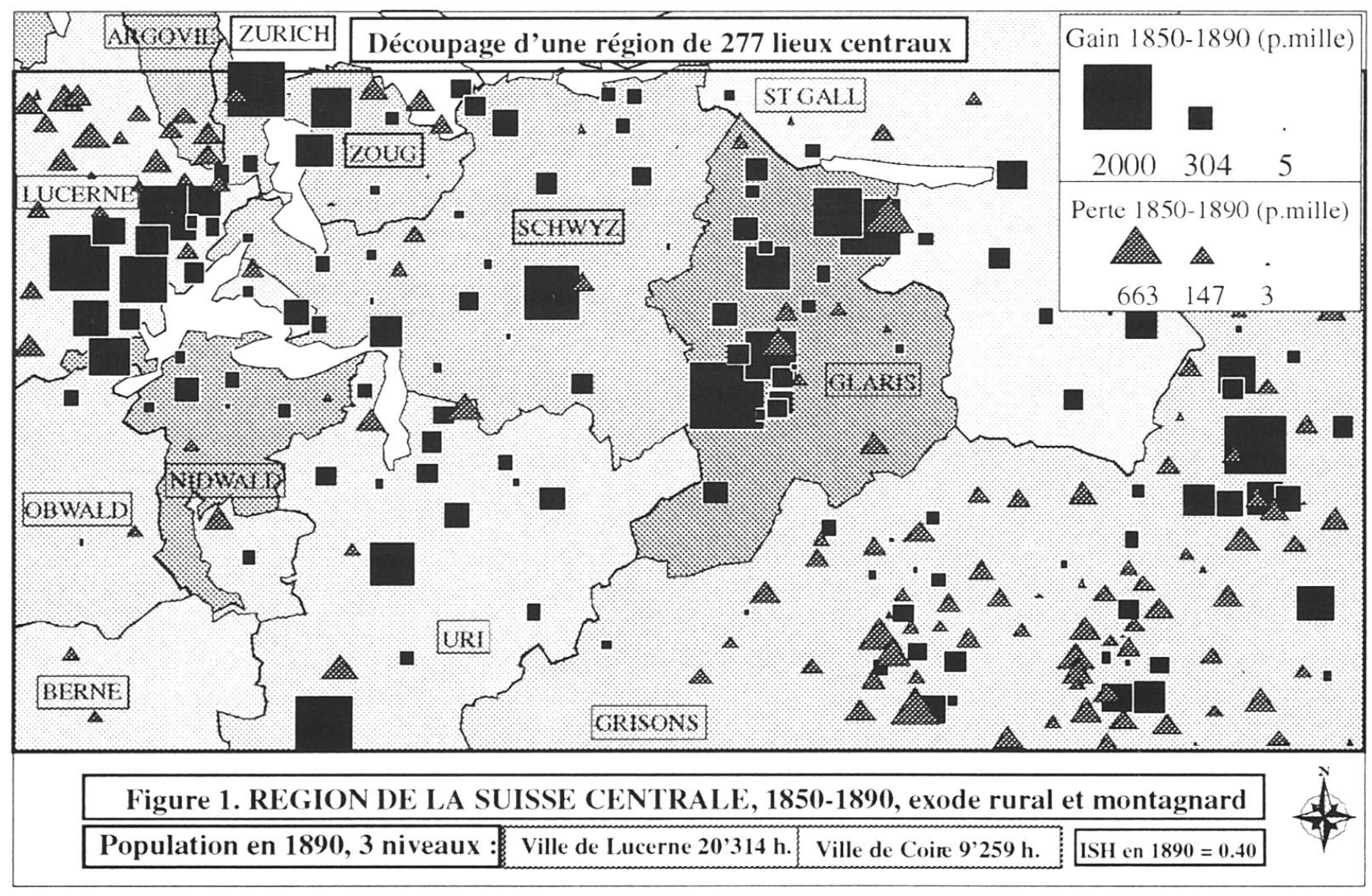




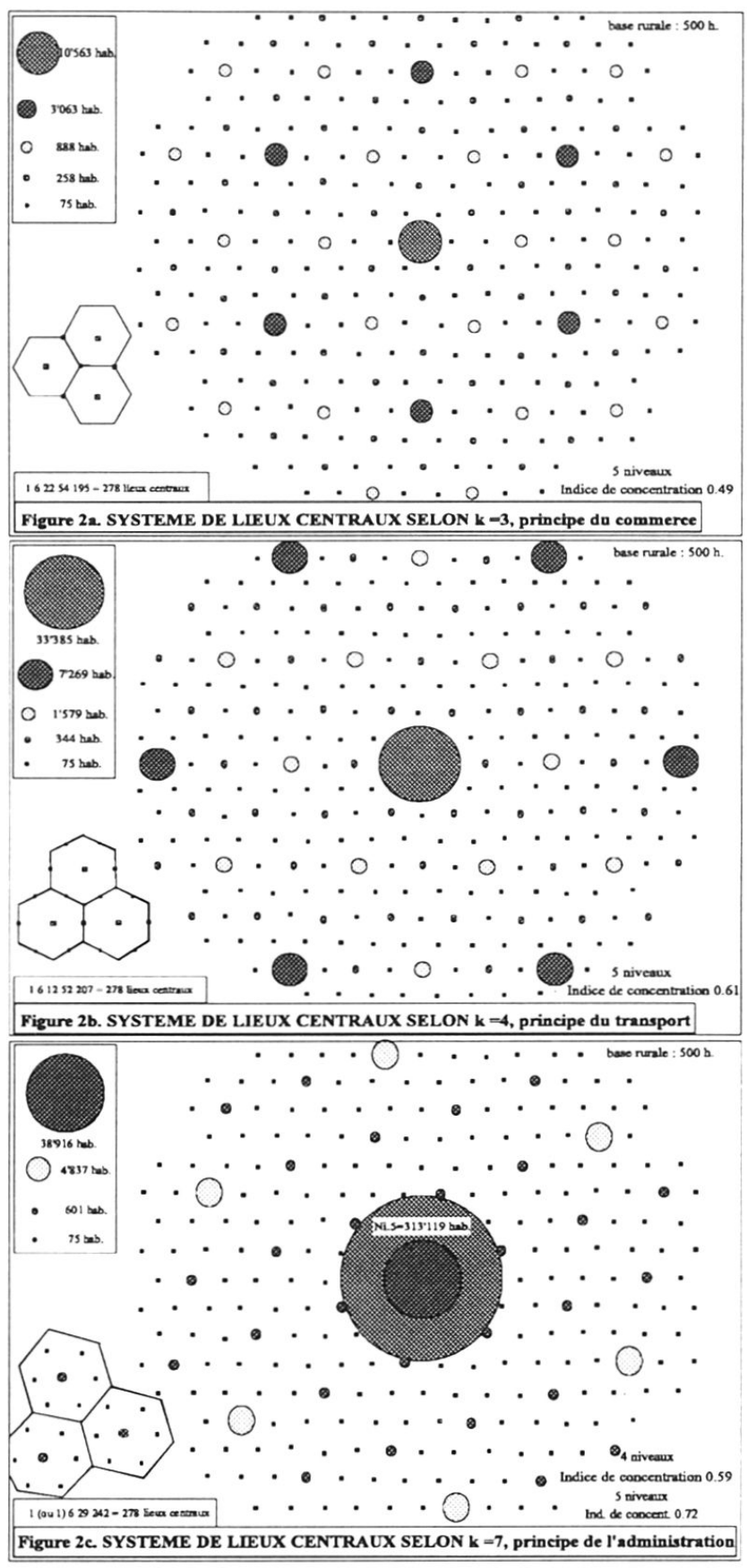

On a donc une structure hiérarchique, à homothétie interne: on pourrait parler de configuration fractale, car le principe régissant un système de lieux centraux est le même à toute échelle et l'ensemble se présente comme une figure scalante: par exemple $\mathrm{k}=3$ (principe du marché rural) signifie 3 dessertes par centre de niveau 2 au niveau 1. Avec $\mathrm{k}=4$, on a 4 dessertes au niveau 1 . Avec $\mathrm{k}=7$, un centre de niveau 2 dessert lui-même et 6 centres de niveau 1. Quelle que soit la taille du système, chacune des trois logiques se reproduit de niveau à niveau. L'exemple proposé pour l'analyse compte 278 lieux centraux ( $F i$ gure $2 a, 2 b, 2 c$. Systèmes de lieux centraux, aspect géométrique). La dimension choisie est évidemment en lien direct avec la taille des sous-ensembles communaux de Suisse que nous nous proposons d'analyser.

2. Beckmann et d'autres établissent un lien entre population rurale/urbaine et hierarchie, calculant des populations théoriques pour chaque niveau. Retenons ici la formule de Beckmann:

$\mathrm{P}_{\mathrm{N}}=\frac{\mathrm{S}(\mathrm{N}-1) * \mathrm{R} * 0.13}{0.87 \mathrm{~N}}$

où $\mathrm{P}=$ population urbaine de niveau $\mathrm{N} ; \mathrm{S}=$ indice $\mathrm{k} ; \mathrm{N}=\mathrm{ni}-$ veau; $R=$ population rurale

La formule peut être retournée afin de calculer, pour une population empirique donnée et une population rurale choisie, le niveau d'un centre. Voici un exemple de calcul des tailles de villes: population urbaine (population rurale $=100$ habitants), avec 7 niveaux: (voir Tableau l)

Un défaut de ce type de formule à un stade d'utilisation empirique réside dans la disposition rigide des effectifs de chaque niveau; on peut supposer, en effet, que les tailles se distribuent régulièrement d'un niveau donné au niveau suivant. Ce fait est illustré par d'autres recherches: Reilly, Zipf et d'autres ont étudié la linéarité rang-taille des villes. Toutefois, le «lissage» statistique des effectifs de

Tableau 1 Tailles de lieux centraux selon Beckmann avec les trois principes $k=3,4,7$

\begin{tabular}{|cc|cc|cc|}
\hline k=3 & Habitants & k=4 & Habitants & k=7 & Habitants \\
ler niveau & 15 & ler niveau & 15 & ler niveau & 15 \\
2e niveau & 52 & 2e niveau & 69 & 2e niveau & 120 \\
3e niveau & 178 & 3e niveau & 316 & 3e niveau & 967 \\
4e niveau & 613 & 4e niveau & 1452 & 4e niveau & 7783 \\
5e niveau & 2113 & 5e niveau & 6677 & 5e niveau & 62624 \\
6e niveau & 7285 & 6e niveau & 30699 & 6e niveau & 503869 \\
7e niveau & 25121 & 7e niveau & 141146 & 7e niveau & 4054122 \\
\hline
\end{tabular}




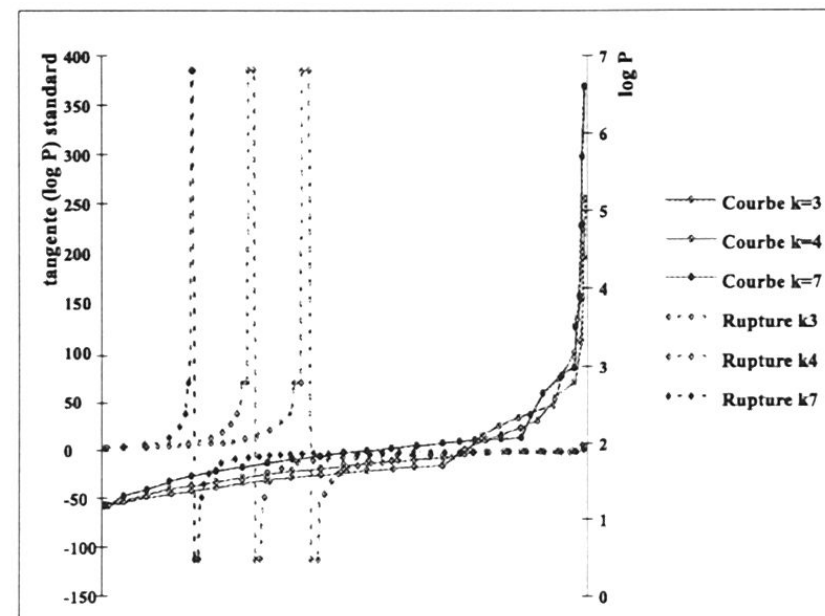

Figure 3. Courbes rang-taille selon Beckmann

population urbaine d'un niveau à un autre, selon le Tableau 1 , ne modifie pas de manière significative les valeurs d'indices (cf. infra. Figure 3. Courbes rang-taille selon Beckmann).

3. Ce passage par la géométrie, puis par le calcul direct des tailles de villes, permet d'envisager un Indice de Structuration Hiérarchique (ISH) qui, statistiquement, se ramène à un indice de concentration (TRICOT, RAFFESTIN, BACHMANN, 1974). Cet indice cumule par itérations (sur machine) les proportions de maillons vides sur un vecteur; le vecteur dispose les effectifs à l'intérieur d'une suite de mailles qui, par exemple, peuvent représenter la superficie habitable d'un territoire urbanisé. Mais on pourrait aussi calculer la concentration des activités tertiaires par rapport à la population des villes. Ici, le calcul d'indices à partir de populations théoriques considère que chaque lieu occupe une superficie unitaire, avec une population rurale constante. En reprenant les effectifs de Beckmann pour des systèmes complets de taille croissante, on obtient ces valeurs d'indices: (voir Tableau 2)

La connaissance du niveau d'un centre complete ainsi la connaissance de l'ISH dans l'étude empirique. Le passage d'une logique à une autre s'opère sur le terrain de manière qualitative et non quantitative: «Les migrations de villes de niveau inférieur vers une ville de niveau supérieur peuvent être commandées par l'apparition dans la ville de niveau supérieur d'une nouvelle activité, ou plus généralement d'une innovation qui conditionne des migrants positivement sélectionnés" (HUSSY, MERCIER, RAFFESTIN, 1985, p. 18).

Seule la taille du systeme modifie la valeur de l'indice d'un territoire obéissant à une même logique avec le même nombre de niveaux. Il est donc nécessaire de fixer cette taille pour pouvoir comparer le modèle à une réalité empirique. On obtient alors les indices suivants: (voir Tableau 3)

Pour des raisons pratiques, l'illustration qui est proposée sur la Figure 2. prend en compte 278 lieux centraux, dont le rang change lorsque le territoire passe d'un principe organisateur à un autre. On voit que pour k=3, le cadre déborde un ensemble complet de cinq niveaux, tandis qu'il est trop petit pour contenir un ensemble de 343 lieux

Tableau 2 Calcul de l'indice de structuration hiérarchique (ISH) sur des systèmes théoriques

\begin{tabular}{|c|c|c|c|c|c|c|c|c|}
\hline $\begin{array}{l}\text { N. Centres } \\
\text { par niveau }\end{array}$ & $k=3$ & ISH & $\begin{array}{l}\text { N. Centres } \\
\text { par niveau }\end{array}$ & $k=4$ & ISH & $\begin{array}{l}\text { N. Centres } \\
\text { par niveau }\end{array}$ & $k=7$ & ISH \\
\hline 96 & \multicolumn{2}{|c|}{ Niveau 1 seul } & 150 & \multicolumn{2}{|c|}{ Niveau 1 seul } & 294 & \multicolumn{2}{|c|}{ Niveau 1 seul } \\
\hline 24 & 1 à 2 & 0.222 & 30 & 1 à 2 & 0.291 & 42 & 1 à 2 & $0.422 *$ \\
\hline 10 & 1 à 3 & 0.342 & 6 & 1 à 3 & $0.422 *$ & 6 & 1 à 3 & 0.579 \\
\hline 1 & 1 à 4 & $0.422 *$ & 1 & 1 à 4 & 0.499 & 1 & 1 à 4 & 0.593 \\
\hline 127 & \multicolumn{2}{|c|}{ lieux centraux } & 187 & \multicolumn{2}{|c|}{ lieux centraux } & 343 & \multicolumn{2}{|c|}{ lieux centraux } \\
\hline
\end{tabular}

" L'indice 0.422 qui réapparaît trois fois illustre un principe d'économie: le niveau supérieur assume la structuration hiérarchique du niveau inférieur, mais à un moindre coût, puisqu'il faut un système à 4 niveaux pour atteindre cet indice avec le principe du marché, tandis qu'il suffit de 3 niveaux pour l'atteindre avec le principe du transport et, avec le principe d'administration, 2 niveaux seulement.

Tableau 3 Indices sur systèmes de même taille

\begin{tabular}{|c|c|c|c|c|c|c|}
\hline ISH & 2 niveaux & 3 niveaux & 4 niveaux & 5 niveaux & 6 niveaux & 7 niveaux \\
\hline$k=3$ & 0.14 & 0.32 & 0.44 & 0.49 & 0.59 & 0.66 \\
\hline$k=4$ & 0.20 & 0.37 & 0.51 & 0.61 & 0.68 & 0.82 \\
\hline$k=7$ & 0.29 & 0.43 & 0.59 & 0.72 & 0.88 & 0.91 \\
\hline
\end{tabular}


sous le principe $k=7$; ceci rapproche le modele des réalités empiriques et il faudra, par la suite, découper des territoires à étudier comptant le même nombre de lieux centraux. Un premier exemple en est fourni par la Figure I. Ce mode d'approche par "fenêtres" implique que certains échantillons couvrent plusieurs systemes dans une logique $\mathrm{k}=3$ (qui totalise 127 centres, cf. Tableau 2), alors que d'autres n'en recouvriront qu'une partie dans une logique $\mathrm{k}=7$ (qui totalise théoriquement 343 centres). Les places centrales étudiées empiriquement seront des communes, dont l'effectif représente pour partie une population rurale et pour partie les habitants du chef-lieu.

\section{Critères empiriques}

L'approche théorique des systèmes territoriaux mobilise, on vient de le voir, trois variables: dimension du système, principe organisateur et nombre de niveaux hiérarchiques; elle doit faire fonctionner ces éléments comme des variables indépendantes car, par exemple, le nombre de lieux considérés dans un système n'a rien à voir avec le nombre de niveaux. La modélisation empirique peut ainsi procéder de diverses manières. Elle peut représenter la suite croissante des lieux centraux, pour en examiner l'allure (premier critère); elle peut aussi mesurer l'ISH du système (second critère) ou encore évaluer le nombre de niveaux (troisième critère). L'étude d'un système urbain dans son évolution historique doit tenter, en définitive, en combinant ces criteres, de retracer les changements successifs du principe organisateur.

La courbe rang-taille obtenue par le logarithme des effectifs de population présente une allure plus ou moins «redressée», c'est-à-dire dont le point d'inflexion (observé sur la dérivée) est plus ou moins proche de l'origine du graphe, indiquant que le système croît à raison d'abord décroissante, puis soudainement à raison croissante, en atteignant les villes de niveau supérieur. Les trois séries calculées par l'équation de Beckmann, avec leurs dérivées, manifestent clairement cette rupture (Figure 3). Celle-ci se déclenche de plus en plus bas dans la hiérarchie quand on passe d'un principe organisateur $\mathrm{k}=3$ à $\mathrm{k}=4$ ou à $k=7$. Il est donc primordial dans l'étude empirique de reconnaitre et comparer ces moments d'inflexion. Ce qui implique l'analyse d'une séquence temporelle de systèmes successifs sur le même espace, si possible à périodicité régulière, ce qui est le cas des recensements suisses. Le cas du bassin lémanique montre ainsi qu'une rupture importante a eu lieu pendant les décennies 1880 à 1910 , manifestee par la perte de population des bourgs ruraux et le renforcement des centres de niveau 3 et 4 ; un second choc se produisant à partir de 1960 (déversement de la population urbaine en périphérie et dépeuplement des montagnes). Figure 4. Le bassin lémanique vers 1880-1910, rupture de $k=3$ vers $k=4$.

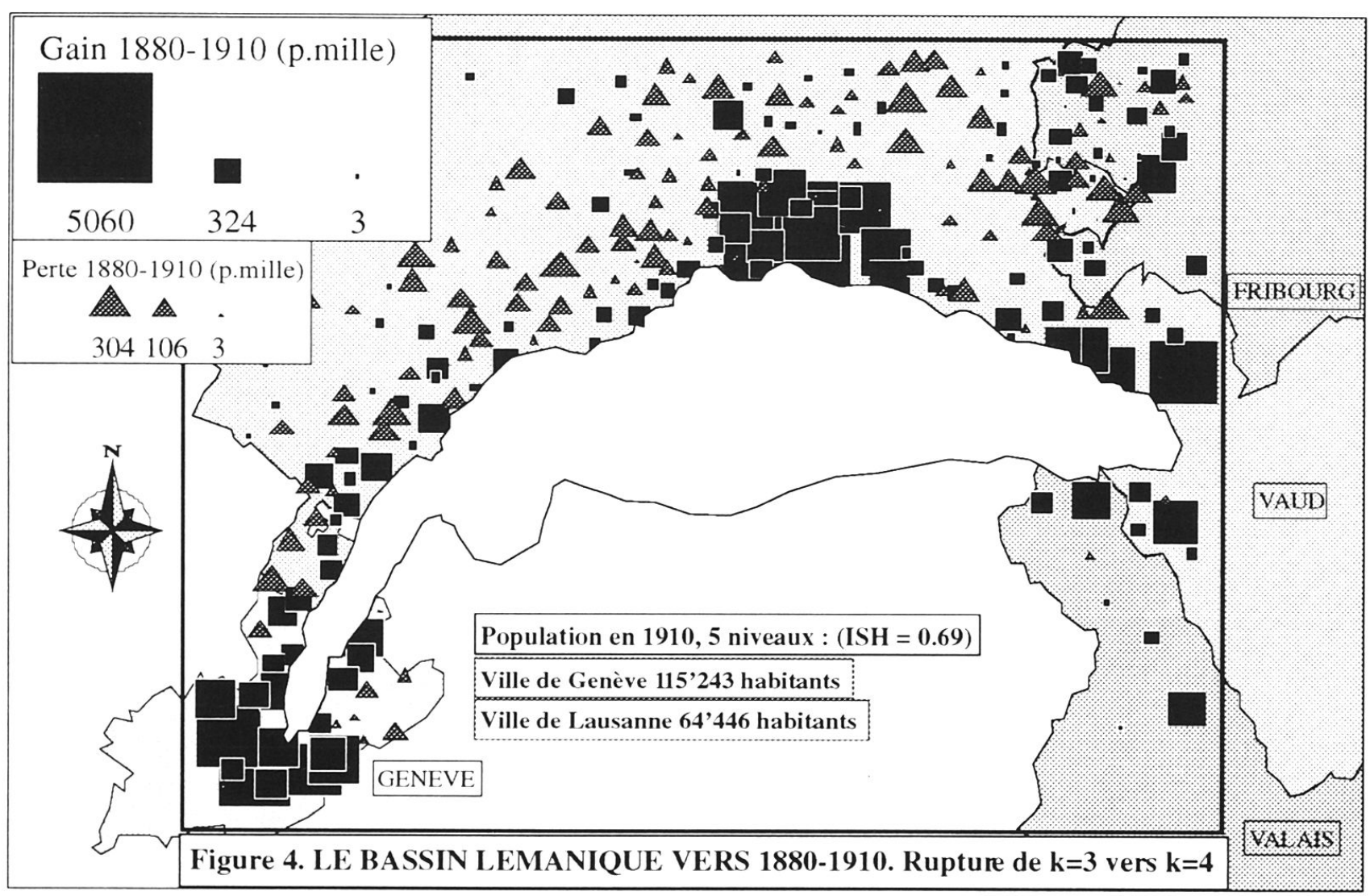


Dans une situation du type décrit dans cet exemple, on observe en effet que la courbe rang-taille s'élève peu à peu, de décennie en décennie, révélant par là une liaison fondamentale entre la taille (croissante) des lieux centraux et le principe organisateur. La pente générale des courbes traduit une structure (cette analogie est fréquemment soulignée: ISARD 1956, BERRY 1967, BEAVON 1977 ) et leurs ruptures révèlent des sous-structures, dont il s'agit de tenir compte pour pouvoir repérer des moments de mutation. Il reste à savoir alors s'il s'agit d'une mutation qualitative (changement de principe organisateur) ou d'un changement du nombre de niveaux hiérarchiques, ou encore des deux phénomènes, qui peuvent être simultanés. Figure 5. Discontinuités des densités de population, Léman.

L'indice de structuration hiérarchique (ISH) obéit précisément au double jeu du principe organisateur $(\mathrm{k})$ et du nombre de niveaux hiérarchiques $(\mathrm{N})$ et doit être ventilé comme l'indique le Tableau 3. Insensible à la taille des lieux centraux si leurs effectifs varient linéairement, par croissance globale, dans le système, l'indice permet en revanche, en inférant la valeur de k, principe organisateur, suite à l'examen chronologique des courbes rang-taille et de leurs ruptures, d’identifier à tout moment le nombre de niveaux du système. Cette méthode est la plus simple; il serait également possible d'évaluer les niveaux par l'examen des effectifs de population pour, ensuite, à l'aide du graphe ISH, trouver le principe k qui régit chaque situation. En tout état de cause, on est amené à superposer les résultats empiriques d'un calcul de l'ISH par décennies aux profils du modèles (établis selon chaque principe organisateur et chaque nombre de niveaux). Un tel graphique (voir la Figure 7) permet d'envisager une prévision par interpolation.

\section{Le système urbain suisse:}

\section{La sortie de l'âge agricole}

L'entité considérée ici comme lieu central sera la commune, dont on ne peut évaluer systématiquement la population rurale. Cependant, les disparités d'effectifs de population entre les communes suisses et le modèle n'entrent pas en ligne de compte, car l'indice est insensible aux transformations linéaires des données, raison pour laquelle on peut négliger l'incidence de la population rurale, considérée comme constante. Notre paramétrage

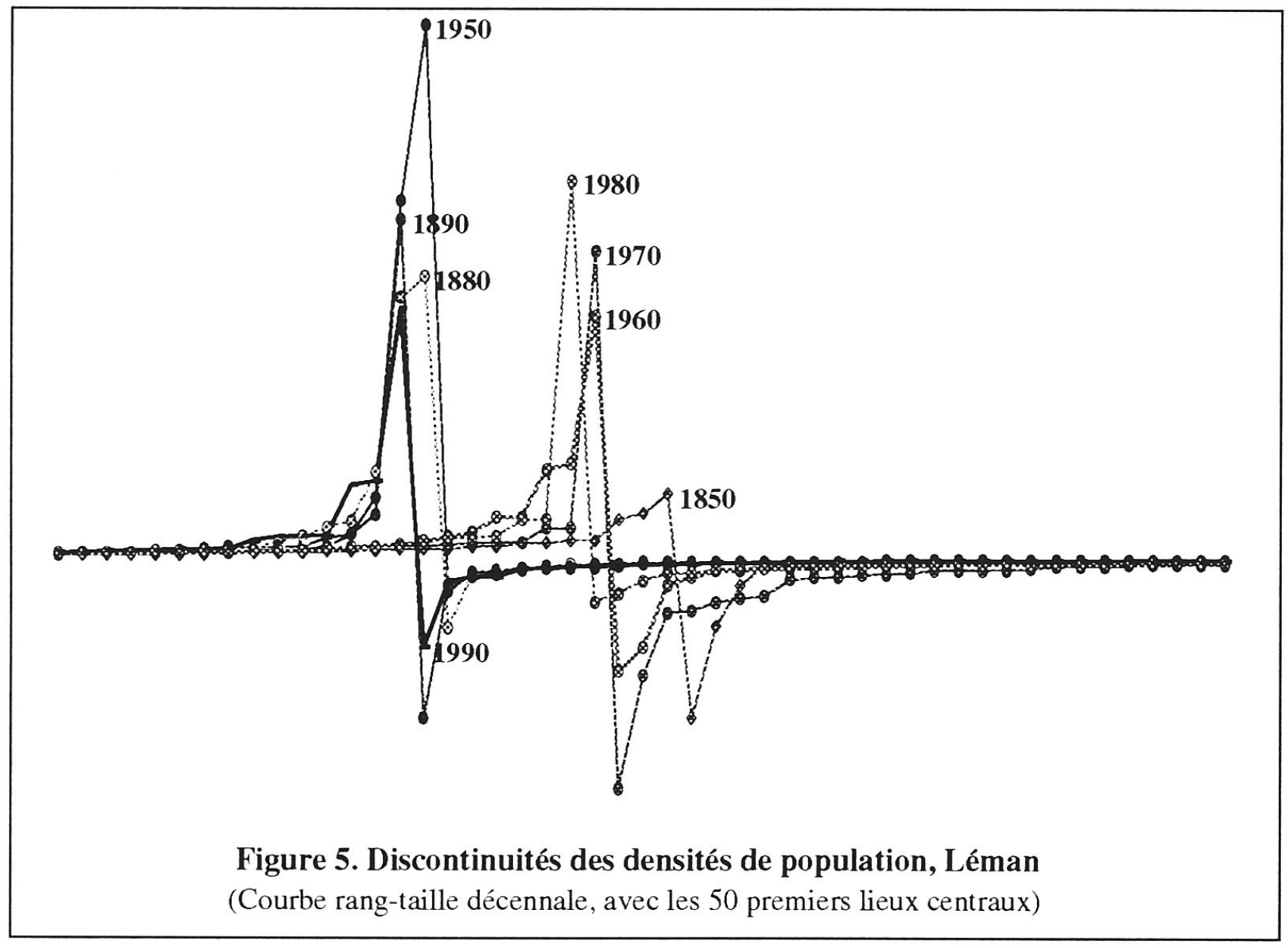




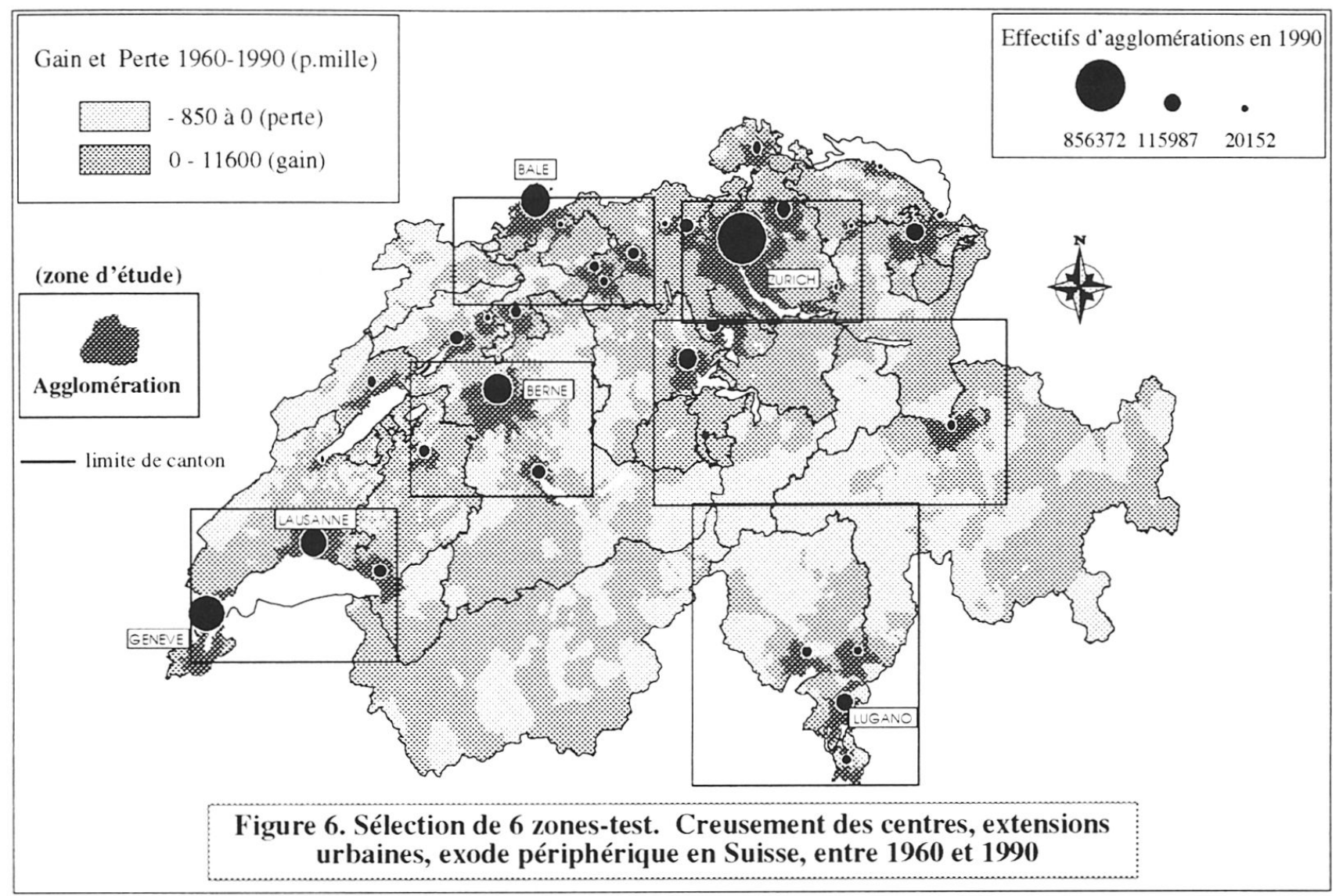

tient compte, en revanche, de la taille des systemes ( 278 lieux centraux). Il s'agit maintenant d'émettre des hypothèses explicatives quant au principe organisateur et au nombre de niveaux. Ce travail peut s'appuyer accessoirement sur l'évolution du nombre d'emplois du tertiaire supérieur (plutôt que des habitants) et l'évolution de l'ISH à partir de ce groupe d'activités. Or il passe, par exemple, pour la Suisse entière, de 0.44 en 1975 à 0.51 en 1985, soit très au-dessus de l'ISH de la population, qu'on le mesure pour toutes les communes, pour les districts ou encore pour les villes d'au moins 1000 habitants.

Les résultats de calcul d'indices sur la Suisse entière par communes ne fournit qu'une indication de tendance: concentration urbaine très impressionnante entre 1850 et 1900, plus lente et régulière jusqu'en 1960-1970; déconcentration au cours des deux dernières décennies, expliquée par l'exode urbain. La confrontation des théories christallériennes et des réalités suisses exige l'adoption d'un échantillonnage régional, opéré autour de villescentres. Afin de tenir un équilibre entre des espaces urbains de plaine et les zones périphériques, le choix s'est porté sur six régions qui regroupent chacune $278 \mathrm{com}$ munes et qui "cadrent» systématiquement l'une ou l'autre agglomération. Voir Figure 6. Sélection de 6 régions de Suisse regroupant 278 communes, et Figure 7. ISH des six régions d'étude.

Les cartes détaillées montrent l'explosion urbaine de la première phase (1850-1900) au détriment de petits cen- tres ruraux qui dépérissent et au profit des centralités nodales, myriade de communes de l'avant-pays en plein dépeuplement; l'explosion, au contraire, des périphéries urbaines au cours d'une troisième période (1960-1990), par formation de réseaux fourmillants autour des villes principales, et qui voit s'abaisser l'ISH; la sélection opérée, désormais, s'avère trop restrictive, car le territoire s'agrandit avec le passage au principe d'administration. La phase intermédiaire (1900-1960) souligne la mise en place des centralités industrielles, avec très peu de déficits communaux d'habitants (augmentation générale des effectifs de population, à savoir des densités et des concentrations). Ainsi, la mutation qualitative n'entraîne pas nécessairement le même type de changement quantitatif. La substitution de rangs dans la hiérarchie, entre un stade primaire et l'émergence du primat de la circulation (1850-1900), a entraîné de fortes baisses de population dans les bourgs ruraux. En revanche, le passage de cette armature urbaine à la base de l'industrialisation, à un nouveau système urbain d'essence tertiaire, à peine commencé, s'est concrétisé par des croissances allométriques des niveaux supérieurs, sans diminution notable d'effectif dans les bourgs déqualifiés.

La dernière décennie accuse une forte déconcentration, avec le creusement des grands centres comme Zurich et Bâle. Le phénomène est général à la Suisse, voire à l'Europe occidentale, car il représente l'évasion démographique hors des centres, suite à un développement du sec- 
teur tertiaire qui, pour les habitants, est synonyme d'une «exurbanisation». La période qui va de 1960 à 1990 voit aussi décliner des centralités de montagne; une nébuleuse de petits accroissements marque, en revanche, les agglomérations jusqu'aux limites de nos cinq fenêtreséchantillons. L'échelle urbaine change, ce qui tendrait à indiquer que l'entité communale ne convient plus à ce stade, ou $\mathrm{k}=7$; il faudra par la suite agréger les communes urbaines en agglomération avant de calculer l'indice (cf. infra). Les fenêtres aménagées appellent les observations suivantes:

1. Le bassin lémanique suisse (Figure 4) offre une comparaison avec la Suisse centrale quant à la première phase de concentration. Au vu des ruptures des courbes rangtaille et des valeurs d'ISH, mais aussi des cartes, on peut affirmer que cette région passe d'une territorialité primaire $(\mathrm{k}=3$, ISH $=0.52$, cinq niveaux $)$ vers 1850 à une logique secondaire (ou industrielle, $\mathrm{k}=4$ ) vers 1880-1990, toujours avec cinq niveaux. Les courbes accusent de nouvelles ruptures après la Seconde Guerre mondiale, bien que celle de 1950 se situe près de celles de la fin du XIXe siècle, alors qu'une série de communes rejoignent la partie «rurale», grâce à un redémarrage agricole, dans les décennies 1960 à 1980. L'ISH culmine en 1970 à 0.76 , en logique d'administration ou tertiaire, encore avec cinq niveaux dominés par Genève, suivie de Lausanne. En 1990, la concentration baisse par diffusion de la centralité, avec seulement quatre niveaux, mais la logique est sans nul doute celle de l'administration. Un effet de littoral explique ce cas extrême d'élévation de la courbe de concentration.

2. Au plus bas des séries ISH se situe la région de la Suisse centrale (cf. Figure I), qui, oscillant entre 0.36 et 0.52 , se transforme au tournant du siècle dans une logique de transport, avec trois niveaux; pour aboutir à une logique tertiaire à partir de 1970, sans fléchissement de la courbe d'indice et encore avec trois niveaux; alors même que des pertes sensibles dans de petits centres ont lieu entre 1960 et 1990. Les ruptures de dérivée proches de l'origine trahissent la nature uniformément contrainte et peu agricole de cette région de montagne.

3 . Une étonnante convergence au cours des trois dernières décennies marque l'évolution des quatre autres régions. Le Tessin se hisse à une logique de transport vers 1930 avec quatre niveaux, puis se stabilise sur celle d'administration durant les deux dernières décennies. La région Berne - Fribourg, fortement rurale jusqu'à la fin du siècle dernier ( $k=3$, cinq niveaux; 1940-50, six niveaux), n'atteint le seuil critique (au vu de sa dérivée) d'une transition vers le secondaire qu'en 1970; seule, la périphérie de Fribourg se développe de manière significative (doublement) entre 1970 et 1990, amorçant du coup le passage à une logique d'administration, orientée vers la Suisse romande. Cet exemple à évolution lente est révélateur d'une forte croissance de population en logique primaire entre 1910 et 1950 .

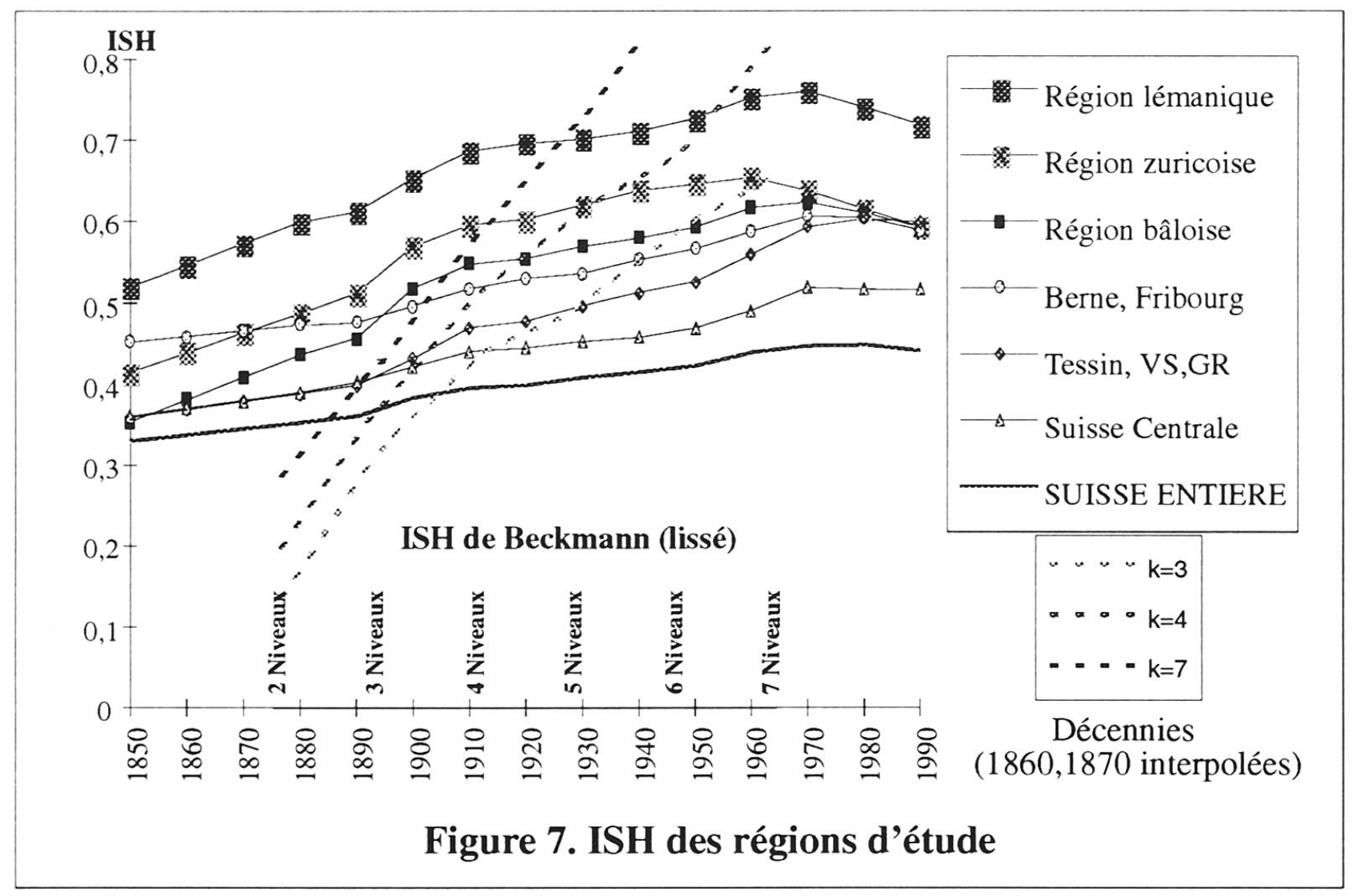


L'agglomération zurichoise opère un parcours semblable, encore que dans d'autres proportions, à celui du Léman; sa population quadruple en 140 ans (de 345000 à 1530000 habitants). La courbe rang-taille n'indique ses seuils de rupture qu'à travers la sous-dérivée, ce qui souligne l'homogénéité urbaine de toute la région. Le fléchissement net de la courbe ISH depuis 1970 manifeste l'inadéquation, comme pour le Léman, du référentiel communal: un accroissement de plus de $50 \%$ s'opere dans quasiment tout le cadre de sélection, entre ce tournant décennal et 1990. Enfin, la région bâloise suit également le profil lémanique; vers 1910 ( $\mathrm{ISH}=0.55$ ), moment auquel la prédominance agricole y céde le pas à l'émergence de la métropole industrielle. Et si les calculs sont perturbés par la position physique marginale de Bâle, le fléchissement de 1970 n'est pas sans lien avec un passage vers une disposition "administrative» de la centralité, avec quatre niveaux.

Cette approche vise à montrer l'intérêt général d'une méthodologie, sans spécifier une problématique régionale particulière. Les fenêtres-échantillons ont pour rôle de souligner des évolutions similaires, mais à rythme différencié; car à elle seule, la carte manifeste les grandes transitions, mais sans les expliquer. Compte tenu de l'abondance des images nécessaires à un suivi des régions période par période, elle gagnerait d'ailleurs à être intégrée (comme c'est notre projet) dans un systeme de visualisation multimédia. Le probleme principal rencontré à ce stade de l'analyse est que l'exercice comparatif reste enfermé dans un système de référence fixe, incompatible avec l'évolution même des territoires. C'est pourquoi, avant d'esquisser une tendance, ce point mérite d'être discuté.

\section{Le changement d'échelle au tournant des années septante}

Il est indéniable que la méthode d'observation et d'analyse présentée jusqu'ici se heurte au paradoxe, manifesté entre réalité empirique et modéle, de la déconcentration (tendance à la baisse de l'ISH) à partir de 1970. A y regarder de près, il semble d'ailleurs que l'origine de ce paradoxe tienne essentiellement à la définition même d'une place centrale. Car, tandis que la statistique utilisée dans notre test emprisonne le calcul de l'ISH, de par sa fixité, l'appréhension conceptuelle de la centralité géographique postule la souplesse et un ajustement périodique de sa définition. Le fait d'avoir adopté la géométrie hexagonale, pour fonder le changement qualitatif de l'armature urbaine, garde toute sa valeur de demonstration; mais l'arithmétique des populations proposée par Beckmann, Parr ou Beguin, utile pour surmonter l'anisotropie de l'espace, trouve ses limites au moment de l'étude empirique dans la longue durée. La taille des villes se modifie au fil des décennies, ce qui remet en cause l'analyse à partir d'un échantillonnage unique.

Qu'est-ce, en effet, qu'une commune suisse au moment de la sortie de l'âge agricole (vers 1850) sinon le noyau élémentaire d'un agrégat de type alvéolaire, compatible avec la logique du marché rural? A l'opposé, la même commune perd toute signification dans un système oủ la centralite domine et ou une ville du plus haut niveau en recouvre une ou plusieurs dizaines d'autres. Physiquement, ces populations ne peuvent s'établir que sur des surfaces aptes à supporter un certain plafond de densité d'habitat. En outre, la logique ou $k=7$ implique d'ellemême un resserrement maximal d'entités urbaines du niveau inférieur, étant toutes desservies par un seul et unique centre, ainsi que de celles qui se situent à des niveaux inférieurs aux précédentes. Ces deux raisons plaident en faveur d'une reformulation de la taille de l'unité empirique, autrement dit, d'une autre géométrie. Géométrie nouvelle qui suppose nécessairement un changement de l'échelle de saisie statistique.

Le probleme est familier des instances administratives d'aménagement du territoire. Ce n'est certes pas sans motif operationnel que les organismes de gestion du territoire éprouvent le besoin de définir périodiquement les nouvelles limites des agglomerations, par certains criteres (population, habitat continu, proportion maximale de population agricole). La nouvelle échelle urbaine de la Suisse depuis les années 1960 est non seulement intercommunale, mais également (au moins en 1990) intercantonale; elle gomme implicitement certaines limites de souverainete. Ainsi surgit subrepticement une territorialité nouvelle. Les relations fonctionnelles entre centres et periphéries nont plus rien à voir avec le maillage communal initial, car le passage a une logique dite d'administration secrete une nouvelle réalite "géostatistique». Dès lors, le géographe est contraint d'adopter un nouveau maillage, pour donner du sens à son modele. Car la transition de $k=3$, par $k=4$, vers $k=7$ dans nos societés occidentales exige la prise en compte d'une configuration de départ et d'une configuration d'arrivée. Un territoire nouveau surgit de la logique nouvelle, non plus seulement par substitution des rôles, c'est-à-dire des rangs dans la hiérarchie, mais également par constitution d'entités urbaines à une autre échelle. Ce fait du recouvrement et de la fusion ressort d'ailleurs graphiquement de la Figure 3 (modele théorique); on ne saurait mieux plaider pour un changement d'échelle.

\section{Le système urbain suisse: \\ 2. L'entrée dans l'ère des régions métropolitaines}

Le nouveau paysage urbain de la Suisse au seuil du prochain millénaire ne saurait donc être traduit par un maillage communal: il ressemble davantage a l'agrégation présentée, selon les critères de 1990: Figure 8. Cette image construite à partir des agglomérations OFS (Office Fédéral de la Statistique) comprend quelques villes isolées et regroupe les périphéries en soldes cantonaux "régionaux"; la superposition du réseau nodal de premier ordre des flux téléphoniques illustre les articulations dynamiques au sein des grandes connexions ur- 


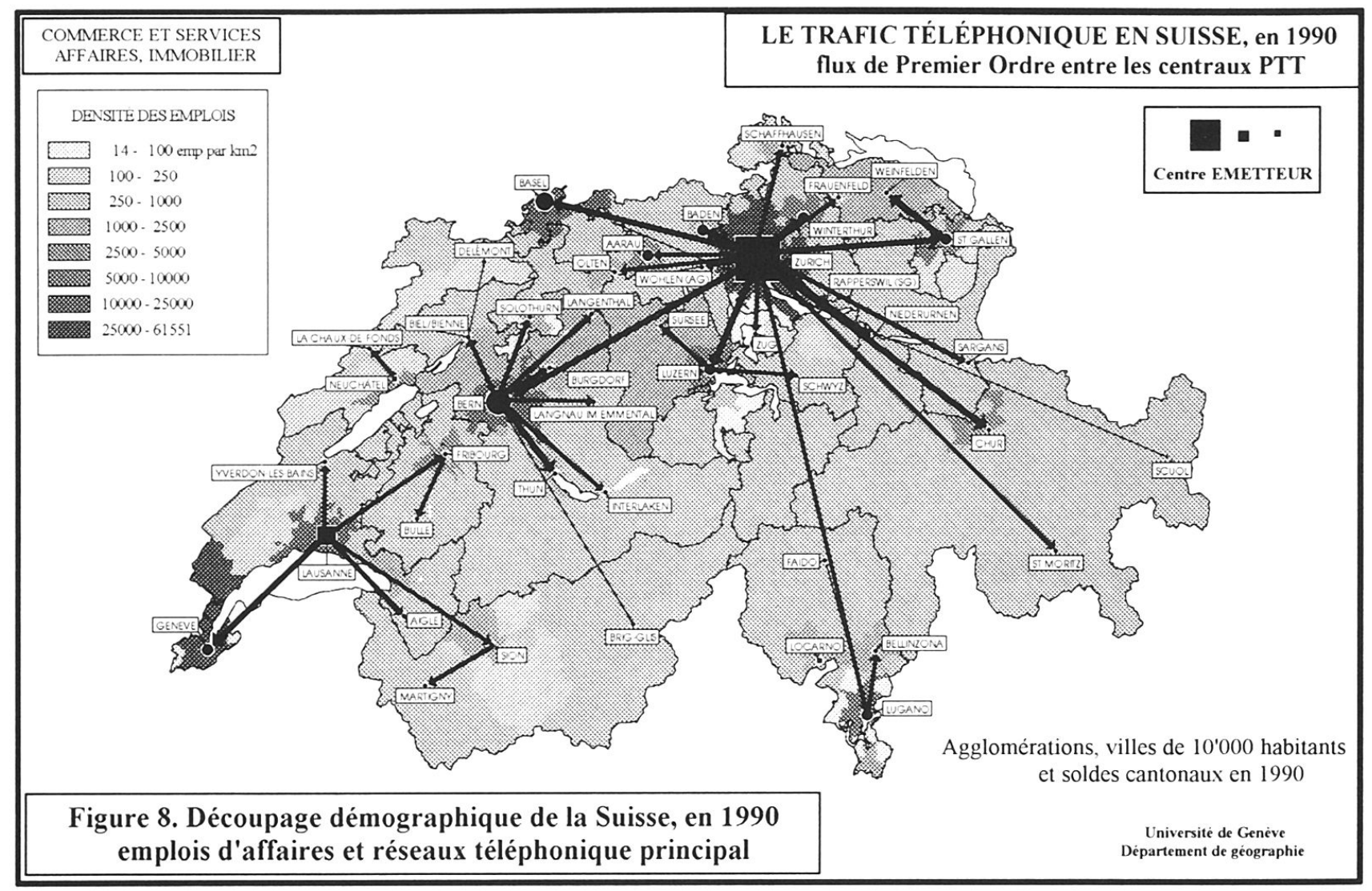

Figure 9. Profil de la Suisse (agglomérations et soldes)

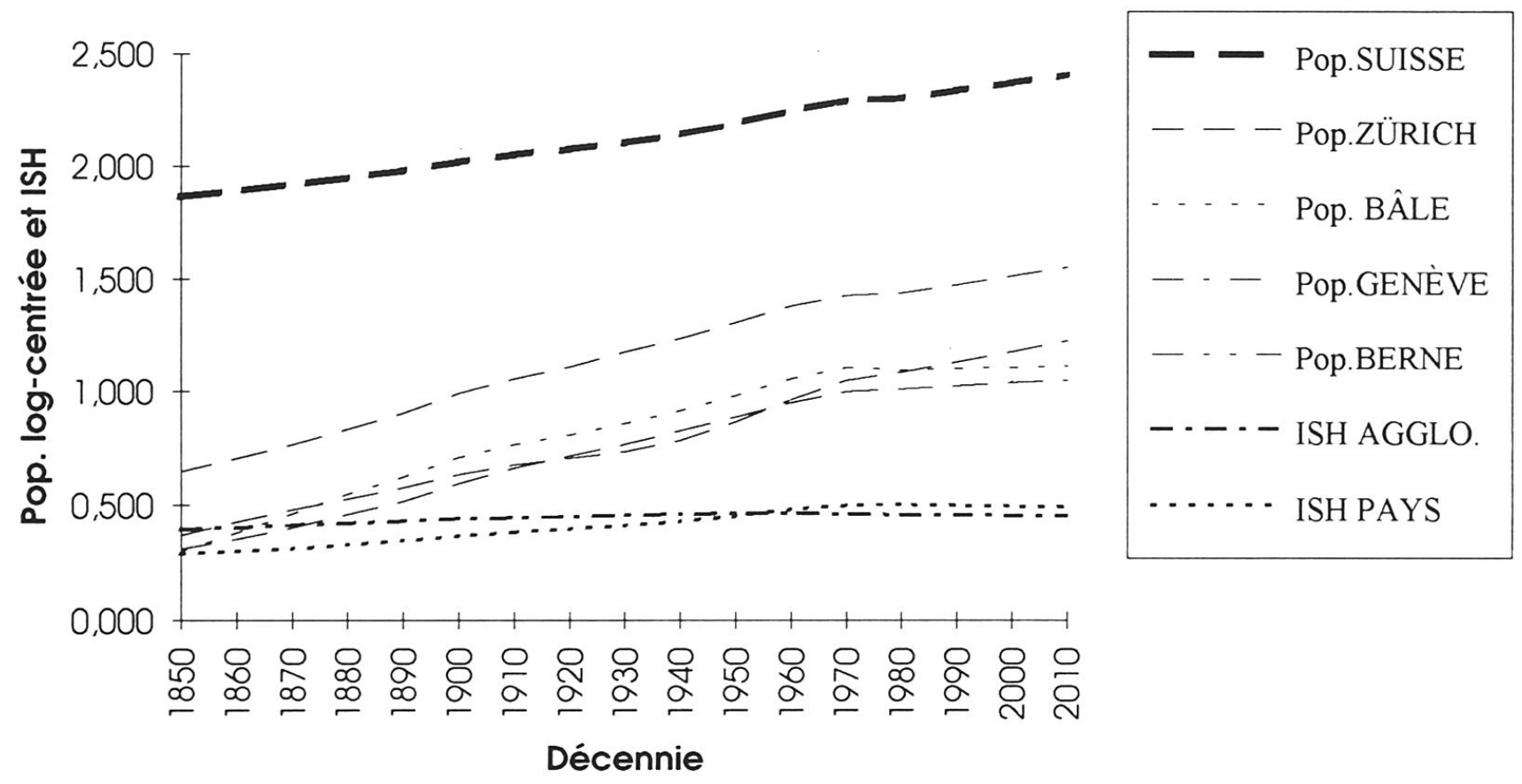


baines. Il est clair que les fenêtres-échantillons ne sont ici plus de mise; rien n'empêche, en revanche, de comparer les courbes diachroniques de l'ISH pour la Suisse entière et pour ses agglomérations, en extrapolant les populations des décennies 2000 et 2010.

Il est sans doute hasardeux de vouloir prédire les effectifs de population des communes à l'horizon 2000 ou 2010. Le comportement naturel est sensible à un environnement économique, pour l'heure, incertain, et le contexte européen ou mondial, qui conditionne les mouvements migratoires à venir, n'est lui-même guère prévisible. La projection la plus réaliste doit considérer des entités géographiques d'une plus grande taille et raisonner à partir d'une évolution globale de la Suisse. Notre modèle en offre un modeste aperçu; car en ajustant une fonction exponentielle du même type aux effectifs par agglomérations, villes isolées et soldes cantonaux, rien n'interdit de leur imputer une croissance constante au rythme des décennies 1980 (pour l'an 2000) et 1990 (pour l'an 2010). Dans cette hypothèse linéaire, on voit que le système urbain suisse voit augmenter ses effectifs de population. En contrepartie, les résultats montrent à nouveau un (très) léger fléchissement de l'ISH, qui passe d'abord de 0.30 en 1850 à 0.50 en 1990, pour plafonner ensuite, en dépit d'une croissance des habitants estimée de $8,3 \%$ pour la décennie en cours et de $8,6 \%$ pour la prochaine décennie. C'est l'irruption, à nouveau, du problème de l'échelle: nul ne saurait prédire les contours des agglomérations à l'horizon 2010 ou 2015. Il est certain pourtant que l'ISH aura repris son allure ascendante, puisque son calcul sera fondé sur de nouvelles extensions des entités urbaines; l'effet obtenu par le passage à l'agglomération, à l'issue de cette expérience, devrait à nouveau se produire. Compte tenu des mutations observées depuis le milieu du XIXe siècle, la croissance touchera les villes en proportion de leur importance actuelle, non de leur effectif, et par diffusion de la centralité. Au vu des correctifs qu'apporte la prise en compte de cette dernière au-delà de 1970, la concentration va se poursuivre; suivant un principe d'allométrie, la logique d'administration doit se renforcer.

On perçoit ainsi la complexité d'une prévision du peuplement, qui s'opère par redimensionnement et non par croissance linéaire: il ne suivra pas, mais dépassera, les tendances observées sur la Figure 9. Cet indice corrige le "tassement» observé entre 1970 et 1990 commune par commune. Il n'est pas entièrement comparable à ceux calculés précédemment, car le nouvel échantillon mêle des périphéries dépeuplées avec les villes isolées, les agglomérations et les centres de premier ordre qui dominent ces dernières. De plus, l'ISH calculé sur les seules agglomérations urbaines est lui aussi décevant, par son amplitude (de 0.40 à 0.50 ) sinon par son mouvement en 160 ans. L'explication se situe dans l'hétérogénéité structurelle de la Suisse à cette échelle, sans qu'un découpage global à tous les niveaux urbains, pour autant, paraisse évident. Par ailleurs, une concentration de 18 grandes villes dans un cadre enserrant la Suisse, d'Aix-les-Bains à Augsbourg et de Milan à Besançon, parmi lesquelles on retient sept villes suisses, donne en 1989 un maigre 0.40 . C'est une hiérarchie complète des villes, et non une sélection drastique ou un agrégat hétérogène, qu'il faudrait pouvoir constituer. En croissance linéaire, la population suisse atteindrait huit millions en 2010; pour la partie septentrionale (région zurichoise, bernoise et bâloise), elle dépasserait à elle seule, en chiffres arrondis, les sept millions. Plutôt cependant que le niveau absolu de ce peuplement, c'est sa modalité, c'est-à-dire sa nature ou sa logique, globalement tertiaire au nord, au sud (Tessin) et à l'ouest lémanique, qui nous semble intéressante et qui doit être retenue pour évaluer ou imaginer, à ce jour en partant d'hier, tous les changements futurs de territorialité.

Références (par ordre chronologique):

CHRISTALLER, W. (1933): Die Zentralen Orte in Süddeutschland. Fischer, Jena (trad. anglaise: Central places in Southern Germany, Englewood Cliffs, Prentice Hall).

BECKMANN, M. J. (1958): City Hierarchies and the Distribution of City Size. Dans: Economic Development and Cultural Change, vol. 6, p. 243-248.

TRICOT, C., RAFFESTIN, C., BACHMANN, D. (1974): Elaboration et construction d'un nouvel indice de concentration. Dans: L'Espace Géographique III(4), p. 303-310.

HUSSY, C. (1980): Genève étude régionale. Essai d'analyse sémiologique en géographie humaine. Thèse, èd. Peter Lang, Berne, p. 97-177.

BEGUIN, H. (1984): The shape of city-size distributions in a central-place system. Dans: Environment and Planning A, p. 749-758.

HUSSY, C., MERCIER, C., RAFFESTIN, C. (1985): Centralité et concentration. Dans: Cahiers de Géographie du Québec, vol. 9, no 76, pp. 9-28.

BLANC, O., HUSSY, C. (1990): Populations et structure des établissements humains. Dans: Nouvelle Géographie de la Suisse, sous la direction de C. Raffestin et J.-B. Racine, Lausanne et Paris, Payot, pp. 197-243.

Logiciel utilisé en cartographie: Cartes et Bases (ADDE). Source des données: Office Fédéral de la Statistique, Berne (Suisse). 